\title{
PRODUCTS OF A COMPACT SPACE AND A METRIC SPACE
}

\author{
YUKINOBU YAJIMA \\ (Communicated by Dennis Burke)
}

\begin{abstract}
Let $X \times Y$ be the product of a compact space $X$ and a metric space $Y$. We consider a continuous closed image $Z$ of $X \times Y$. Moreover, we consider a closed subspace $R$ in $X \times Y$ which is a neighborhood retract of it. It is proved in this paper that $Z$ (respectively, $R$ ) is a Lasnev (metrizable) space iff all compact subspaces of $Z(R)$ are metrizable.
\end{abstract}

1. Introduction. Klebanov $\left[\mathbf{K}_{1}\right]$ proved that a continuous closed image $Z$ of the product of a generalized Cantor cube $D^{\tau}$ and a metric space $Y$ is a Lašnev space iff each dyadic compact subspace of $Z$ is metrizable. Showing that the " $D^{\tau}$ " can be replaced by "a compact space $X$," we obtain the first theorem. On the other hand, Tsuda $[\mathbf{T}]$ has recently proved that a nonmetrizable $\operatorname{ANR}(P)$ contains a copy of the Tychonoff cube $I^{\omega_{1}}$ of uncountable weight, where $P$ is the class of all paracompact $p$-spaces. This result is a nice generalization of Šepin's $\left[\mathbf{S}_{1}\right]$. The second theorem is a further generalization of Tsuda's result. These theorems are stated in $\S 4$.

In $\S 2$, we deal with maps from subspaces of products. Many results concerning the question of when such maps depend on countably many coordinates have been obtained (for example, see the list in [H, p. 27]). However, we give a result which seems to be new. In $\S 3$, using this result, we prove a structural lemma. It is not difficult from this lemma to derive our main theorems.

All spaces are assumed to be Hausdorff. Without special mention, all maps are assumed to be continuous and onto. However, pay attention that maps which are into appear occasionally. For a map $f: X \rightarrow Y$ and a subspace $A$ of $X$, we denote by $f \mid A$ the restriction of $f$ to $A$.

2. Maps from subspaces of products. Let $S_{\lambda}, \lambda \in \Lambda$, be spaces. Consider the product $S=\prod_{\lambda \in \Lambda} S_{\lambda}$. For each $\Gamma \subset \Lambda, \pi_{\Gamma}$ denotes the projection from $S$ onto $\prod_{\lambda \in \Gamma} S_{\lambda}$. We say that $U=\prod_{\lambda \in \Lambda} U_{\lambda} \subset S$ is a basic open set in $S$ if each $U_{\lambda}$ is open in $S_{\lambda}$ and $U_{\lambda} \neq S_{\lambda}$ only for finitely many $\lambda \in \Lambda$. Note that each basic open set $U$ in $S$ satisfies $\pi_{\xi}^{-1} \pi_{\xi}(U)=U$ for some finite $\xi \subset \Lambda$.

Let $X$ be a subspace of $S=\prod_{\lambda \in \Lambda} S_{\lambda}$ and $T$ a space. Recall that a map $f: X \rightarrow T$ is said to depend on countably many coordinates (cf. $[\mathbf{H}]$ ) if there exists a countable subset $\Gamma$ of $\Lambda$ such that $f(x)=f\left(x^{\prime}\right)$ whenever $x, x^{\prime} \in X$ and $\pi_{\Gamma}(x)=\pi_{\Gamma}\left(x^{\prime}\right)$.

Received by the editors January 23, 1986 and, in revised form, April 11, 1986 and August 11, 1986.

1980 Mathematics Subject Classification (1985 Revision). Primary 54B10, 54C05, 54E35; Secondary $54 \mathrm{C} 10,54 \mathrm{C} 15$.

Key words and phrases. Compact space, metric space, product, closed map, perfect map, Lasnev space, $G_{\delta}$-diagonal, neighborhood retract. 
Secondly, recall that a space $T$ has a $G_{\delta}$-diagonal if there exists a sequence $\left\{\mathcal{H}_{i}\right\}$ of open covers of $T$ such that

$$
\bigcap_{i<\omega} \operatorname{St}\left(t, \mathscr{H}_{i}\right)=\{t\} \quad \text { for each } t \in T
$$

where $\operatorname{St}\left(t, \mathscr{H}_{i}\right)=\bigcup\left\{H \in \mathscr{H}_{i}: t \in H\right\}$. Such a sequence $\left\{\mathscr{H}_{i}\right\}$ is called a $G_{\delta^{-}}$diagonal sequence of $T$. Let us note that each compact subspace of a space with a $G_{\delta^{-}}$ diagonal is metrizable.

Now, we give the following proposition, which plays an important role in the proof of the Structural Lemma stated in the next section.

Proposition. Let $S_{\lambda}, \lambda \in \Lambda$, and $T$ be spaces. Let $X$ be a subspace of the product $\prod_{\lambda \in \Lambda} S_{\lambda}$. If $X$ is Lindelöf and $T$ has a $G_{\delta}$-diagonal, then every map $f: X \rightarrow T$ depends on countably many coordinates.

Proof. Let $\left\{H_{i}\right\}$ be a $G_{\delta}$-diagonal sequence of $T$. For each $i<\omega$, we take a collection $\mathcal{G}_{i}$ of basic open sets in $\prod_{\lambda \in \Lambda} S_{\lambda}$ such that $\mathcal{G}_{i}$ covers $X$ and for each $G \in \mathcal{G}_{i}$ there exists some $H \in \mathscr{H}_{i}$ with $G \cap X \subset f^{-1}(H)$. Since $X$ is Lindelöf, one can choose a countable subcollection $\left\{G_{i j}: j<\omega\right\}$ of $\mathcal{G}_{i}$ such that $X \subset \bigcup_{j<\omega} G_{i j}$. For each $i, j<\omega$, take a finite subset $\xi_{i j}$ of $\Lambda$ such that $\pi_{\xi_{i j}}^{-1} \pi_{\xi_{i j}}\left(G_{i j}\right)=G_{i j}$. Put $\Gamma=\bigcup_{i, j<\omega} \xi_{i j}$. Clearly, $\Gamma$ is countable. Now, pick any points $x, x^{\prime} \in X$ with $\pi_{\Gamma}(x)=\pi_{\Gamma}\left(x^{\prime}\right)$. For each $i<\omega$, one can take some $j_{i}<\omega$ such that $x \in G_{i j_{i}} \in \mathcal{G}_{i}$. Then we have

So it follows that

$$
x^{\prime} \in \pi_{\Gamma}^{-1} \pi_{\Gamma}(x) \subset \pi_{\Gamma}^{-1} \pi_{\Gamma}\left(G_{i j_{i}}\right) \subset G_{i j_{i}}
$$

$$
\begin{aligned}
\left\{f(x), f\left(x^{\prime}\right)\right\} & \subset \bigcap_{i<\omega} f\left(G_{i j_{i}} \bigcap X\right) \\
& \subset \bigcap_{i j_{i}} \operatorname{St}\left(f(x), \nvdash_{i}\right)=\{f(x)\} .
\end{aligned}
$$

This means $f(x)=f\left(x^{\prime}\right)$. The proof is complete.

3. Factoring maps from products. In this section, a map which is not assumed continuous is called a function. That is, a map is a continuous function. The following fact is well-known.

FACT. Let $A, B$, and $C$ be spaces. Let $f: A \rightarrow B, g: A \rightarrow C$, and $h: C \rightarrow B$ be functions such that $f=h \circ g$.

(1) If $f$ is a (closed) map and $g$ is a quotient map, then $h$ is a (closed) map.

(2) If $g$ and $h$ are maps and $f$ is a perfect map, then $g$ and $h$ are perfect maps.

In the proofs of $\left[\mathbf{K}_{1}\right.$, Theorem 1.1] and [ $\mathbf{K}_{2}$, Theorem 7$]$, Klebanov proved the following:

Let $C=\prod_{\lambda \in \Lambda} C_{\lambda}$ be a product of compact metric spaces and $Y_{0}$ a metric space with $\operatorname{dim} Y_{0}=0$. If a map $f: C \times Y_{0} \rightarrow Z$ satisfies that $f(C \times\{y\})$ is metrizable for each $y \in Y_{0}$, then there exist a metric space $M$, a perfect map $g: C \times Y_{0} \rightarrow M$, and a map $h: M \rightarrow Z$ such that $f=h \circ g$.

Our Structural Lemma below is an extension of this factorization. The proof of it is a modification of Klebanov's. However, we adopt the style of the proof of [ $\mathbf{T}$, Theorem] instead of his. 
StRUCTURAL LEMMA. Let $X$ be a compact space and $Y_{0}$ a metric space with $\operatorname{dim} Y_{0}=0$. Let $G$ and $F$ be an open set and a closed set in $X \times Y_{0}$, respectively, with $F \subset G$. If a map $f: G \rightarrow Z$ satisfies that $f(G \cap X \times\{y\})$ has a $G_{\delta}$-diagonal for each $y \in Y_{0}$, then there exist a metric space $M$, a perfect map $g: F \rightarrow M$, and a map $h: M \rightarrow f(F)$ such that $f \mid F=h \circ g$.

PROOF. Since $X$ is compact, we can regard $X$ as a closed subspace of a Tychonoff cube $\prod_{\lambda \in \Lambda} I_{\lambda}$, where each $I_{\lambda}$ is a copy of the unit interval $[0,1]$. Moreover, we may assume without loss of generality that $G$ is an open $F_{\sigma}$-set in $X \times Y_{0}$ and that $G_{y}=G \cap X \times\{y\}$ is nonempty for each $y \in Y_{0}$. Since $G_{y}$ is an $F_{\sigma}$-set in $X \times\{y\}$, it is Lindelöf ( $\sigma$-compact). It follows from the Proposition that $f \mid G_{y}$ depends on countably many coordinates. That is, for each $y \in Y_{0}$, there exists a countable subset $\Gamma(y)$ of $\Lambda$ such that

(1) $(x, y),\left(x^{\prime}, y\right) \in G$ and $\pi_{\Gamma(y)}(x)=\pi_{\Gamma(y)}\left(x^{\prime}\right)$ imply $f(x, y)=f\left(x^{\prime}, y\right)$.

By the assumption of $Y_{0}$, it has a base $\mathcal{V}=\bigcup_{i<\omega} \mathcal{V}_{i}$ such that for each $i<\omega$

(2) $V_{i}$ is a discrete clopen cover of $Y_{0}$,

(3) $\sup \left\{\operatorname{diam} V: V \in \mathcal{V}_{i}\right\}<1 / i$,

(4) $V_{i+1}$ refines $V_{i}$

For each $y \in Y_{0}$, observe that one can uniquely choose the increasing sequence $\left\{V_{i}\right\}$ such that $y \in V_{i} \in \mathcal{V}_{i}$ for each $i<\omega$. For each $V \in \mathcal{V}$, assign a point $y_{V} \in V$. Moreover, for each $V \in \mathcal{V}$, one can take a countable subset $\Gamma(V)$ of $\Lambda$ such that

(5) $\Gamma\left(y_{V}\right) \subset \Gamma(V)$,

(6) $\Gamma\left(V_{1}\right) \subset \Gamma\left(V_{2}\right) \subset \cdots$ if $y \in V_{i} \in \mathcal{V}_{i}$ for each $i<\omega$.

Put $I(V)=\prod_{\lambda \in \Gamma(V)} I_{\lambda}$. Put $M_{i}=\bigoplus\left\{I(V): V \in \mathcal{V}_{i}\right\}$ for each $i<\omega$, where $\bigoplus$ denotes the topological sum. Since $M_{i}$ is a metric space, so is $\prod_{i<\omega} M_{i}$. For each $i<\omega$, we take a function $\phi_{i}: G \rightarrow M_{i}$ defined by

$$
\phi_{i}(x, y)=\pi_{\Gamma\left(V_{i}\right)}(x) \in I\left(V_{i}\right) \subset M_{i} \quad \text { for each }(x, y) \in G,
$$

where $y \in V_{i} \in V_{i}$. Then it is easily seen that each $\phi_{i}$ is continuous (and into). Moreover, take a map $\tilde{g}: G \rightarrow \prod_{i<\omega} M_{i}$ (which is into) defined by $\tilde{g}(x, y)=$ $\left\{\phi_{i}(x, y)\right\}$ for each $(x, y) \in G$. Put $\Delta(y)=\bigcup_{i<\omega} \Gamma\left(V_{i}\right)$ for each $y \in Y_{0}$, where $y \in V_{i} \in \mathcal{V}_{i}$ for each $i<\omega$. Then it follows from (3) and the choices of $\phi_{i}$ and $\tilde{g}$ that

(7) $\tilde{g}(x, y)=\tilde{g}\left(x^{\prime}, y^{\prime}\right)$ iff $(x, y),\left(x^{\prime}, y^{\prime}\right) \in G, y=y^{\prime}$, and $\pi_{\Delta(y)}(x)=\pi_{\Delta(y)}\left(x^{\prime}\right)$.

Now, we put $M=\tilde{g}(F)$ and $g=\tilde{g} \mid F$. Clearly, $M$ is a metric space and $g: F \rightarrow M$ is a map. We show that

(8) $g$ is a perfect map.

Pick any $(x, y) \in F$. By (7), we have

$$
g^{-1} g(x, y)=\tilde{g}^{-1} \tilde{g}(x, y) \cap F=\left(\pi_{\Delta(y)}^{-1} \pi_{\Delta(y)}(x) \times\{y\}\right) \cap F .
$$

Since $F$ is closed in $X \times Y_{0}, g^{-1}(x, y)$ is compact. Next, take any open set $U$ in $F$ such that $g^{-1} g(x, y) \subset U$. It suffices to show that there exists an open neighborhood $B$ of $g(x, y)$ in $M$ such that $g^{-1}(B) \subset U$. Assume the contrary. Then one can choose a sequence $\left\{\left(x_{n}, y_{n}\right)\right\}$ of points in $F \backslash U$ such that $\left\{g\left(x_{n}, y_{n}\right)\right\}$ converges to $g(x, y)$ in $M$. Moreover, we may assume that $\left\{y_{n}\right\}$ converges to $y$ in $Y_{0}$. Since $X$ is compact, $\left\{x_{n}\right\}$ has a cluster point $x_{0}$ in $X$. Hence $\left\{\left(x_{n}, y_{n}\right)\right\}$ has the cluster point $\left(x_{0}, y\right)$ in $X \times Y_{0}$. Since $F \backslash U$ is closed in $X \times Y_{0}$, we have $\left(x_{0}, y\right) \in F \backslash U$. By the continuity of $g,\left\{g\left(x_{n}, y_{n}\right)\right\}$ has the cluster point $g\left(x_{0}, y\right)$ 
in $M$. This means $g\left(x_{0}, y\right)=g(x, y)$. Thus, we get $\left(x_{0}, y\right) \in g^{-1} g(x, y) \subset U$. This contradiction to $\left(x_{0}, y\right) \notin U$ establishes (8).

Let us observe that

(9) $g(x, y)=g\left(x^{\prime}, y^{\prime}\right)$ imples $f(x, y)=f\left(x^{\prime}, y^{\prime}\right)$.

Indeed, for each $i<\omega$, take $V_{i} \in V_{i}$ with $y \in V_{i}$ and let $y_{i}=y_{V_{i}}$. By $\tilde{g}(x, y)=$ $\tilde{g}\left(x^{\prime}, y^{\prime}\right),(5)$, and $(7)$, we have $(x, y),\left(x^{\prime}, y^{\prime}\right) \in G, y=y^{\prime}$, and $\pi_{\Gamma\left(y_{i}\right)}(x)=\pi_{\Gamma\left(y_{i}\right)}\left(x^{\prime}\right)$ for each $i<\omega$. Since $\left\{y_{i}\right\}$ converges to $y$ and $G$ is open in $X \times Y_{0},\left\{\left(x, y_{i}\right)\right\}$ and $\left\{\left(x^{\prime}, y_{i}\right)\right\}$ are eventually in $G$. By $(1)$, we have $f\left(x, y_{i}\right)=f\left(x^{\prime}, y_{i}\right)$ except finitely many $i$ 's. Hence one can conclude $f(x, y)=f\left(x^{\prime}, y\right)=f\left(x^{\prime}, y^{\prime}\right)$ because $f$ is a map.

It follows from (9) that a function $h: M \rightarrow f(F)$ can be defined such as $f \mid F=$ $h \circ g$. By (8), $g$ is quotient. Hence it follows from Fact (1) that $h$ is a map. The proof of the Structural Lemma is complete.

4. Main results. Recall that a Lašnev space is a continuous closed image of a metric space. For a space $X$, we denote by $1_{X}$ the identity map on $X$.

THEOREM 1. Let $X$ be a compact space and $Y$ a metric space. Let $Z$ be a continuous closed (perfect) image of the product $X \times Y$. Then the following are equivalent.

(a) $Z$ is a Lašnev (metrizable) space.

(b) Each compact subspace of $Z$ is metrizable.

(c) Each continuous image of $X$ into $Z$ is metrizable.

PROOF. The implications $(\mathrm{a}) \rightarrow(\mathrm{b}) \rightarrow(\mathrm{c})$ are obvious.

(c) $\rightarrow(\mathrm{a})$ : Since $Y$ is a metric space, there exist a metric space $Y_{0}$ with $\operatorname{dim} Y_{0}=0$ and a perfect map $\phi: Y_{0} \rightarrow Y$. Let $\psi: X \times Y \rightarrow Z$ be a closed (perfect) map. Considering $F=G=X \times Y_{0}$ and $f=\psi \circ\left(1_{X} \times \phi\right)$ in the Structural Lemma, it assures the existences of a metric space $M$, a perfect map $g: X \times Y_{0} \rightarrow M$ and a map $h: M \rightarrow Z$ such that $f=h \circ g$. It follows from Fact (1) (and (2)) that $h$ is a closed (perfect) map. Hence $Z$ is a Lašnev (metrizable) space.

REMARK 1. Since dyadic compact space means a continuous image of a generalized Cantor cube $D^{\tau},\left[\mathbf{K}_{1}\right.$, Theorem 1.1] is an immediate consequence of Theorem 1.

REMARK 2. A space $Z$, each compact subspace of which is metrizable, is characterized as a continuous compact-covering image of a metric space (cf. [MN, Theorem 1.1]).

Recall that a subspace $R$ of a space $S$ is called a neighborhood retract of $S$ if there exist an open set $U$ in $S$ with $R \subset U$ and a map $r: U \rightarrow R$, called a retraction, such that $r \mid R=1_{R}$. A space $S$ is said to be $\sigma$-metrizable if $S$ can be represented as a countable union of closed metrizable subspaces.

THEOREM 2. Let $X$ be a compact space and $Y$ a metric space. Let $R$ be a closed subspace which is a neighborhood retract of the product $X \times Y$. Then the following are equivalent.

(a) $R$ is metrizable.

(b) Each compact subspace in $R$ is metrizable.

(c) Each continuous image of an open $F_{\sigma}$-set in $X$ into $R$ is $\sigma$-metrizable.

ProOF. (a) $\rightarrow$ (b): Obvious. 
(b) $\rightarrow$ (c): Let $A$ be an open $F_{\sigma}$-set in $X$ and $A^{\prime}$ a continuous image of $A$ into $R$. Snce $A$ is $\sigma$-compact, so is $A^{\prime}$. Hence $A^{\prime}$ is $\sigma$-metrizable.

(c) $\rightarrow$ (a): By the assumption, there exist an open set $W$ in $X \times Y$ with $R \subset W$ and a retraction $r: W \rightarrow R$. We may assume that $W$ is an $F_{\sigma}$-set in $X \times Y$. One can take a metric space $Y_{0}$ with $\operatorname{dim} Y_{0}=0$ and a perfect map $\phi: Y_{0} \rightarrow Y$. Let $p=1_{X} \times \phi$. Moreover, let $G=p^{-1}(W), F=p^{-1}(R)$, and $f=r \circ(p \mid G)$. Since a $\sigma$-metrizable space has a $G_{\delta}$-diagonal, by (c), $f(G \cap X \times\{y\})$ has a $G_{\delta}$-diagonal for each $y \in Y_{0}$. It follows from the Structural Lemma that there exist a metric space $M$, a perfect map $g: F \rightarrow M$, and a map $h: M \rightarrow R$ such that $f \mid F=h \circ g$. Since $f|F=r \circ(p \mid F)=p| p^{-1}(R)$ and $p$ is a perfect map, $f \mid F$ is also a perfect map. By Fact (2), $h$ is a perfect map. Therefore $R$ is metrizable.

Let $P$ denote the class of all paracompact $p$-spaces.

COROLlaRY (TSUDA $[\mathbf{T}]$ ). Each nonmetrizable ANR(P) contains a copy of Tychonoff cube $I^{\omega_{1}}$ of uncountable weight.

ProOF. Let $P$ be a nonmetrizable $\operatorname{ANR}(P)$. Note that each paracompact $p$ space can be regarded as a closed subspace of the product $I^{\tau} \times Y$ of a Tychonoff cube $I^{\tau}$ and metric space $Y$. So $P$ is a closed subspace of the product $I^{\tau} \times Y$ and is a neighborhood retract of it. It follows from Theorem 2 that there exists some continuous image of an open $F_{\sigma}$-set in $I^{\tau}$ into $P$ such that the image is not $\sigma$-metrizable. Since an open $F_{\sigma}$-set in $I^{\tau}$ is the countable union of Tychonoff cubes $I^{\tau}$ 's, $P$ contains some nonmetrizable generalized Peano continuum $C$ (i.e., a continuous image of some Tychonoff cube). It follows from $\left[\mathbf{S}_{2}\right.$, Corollary 1$]$ that $C$ contains a copy of $I^{\omega_{1}}$.

As stated above, each paracompact $p$-space can be considered as a closed subspace of some product $X \times Y$ of a compact space $X$ and a metric space $Y$. So, it is natural to ask whether "the product $X \times Y$ " in Theorem 1 can be replaced by "a closed subspace $R$ in $X \times Y$." Moreover, it is also natural to ask whether "neighborhood retract" in Theorem 2 can be excluded. However, the answers to these questions are both negative by the following [MN, Example 5.2]: There exists a nonmetrizable paracompact p-space, each compact subspace of which is metrizable.

Finally, the author wish to thank H. Ohta, Y. Tanaka, T. Terada, and K. Tsuda for their helpful suggestions and advice.

\section{REFERENCES}

[M.] Husek, Continuous mapping on subspaces of products, Conf. Rings of Continuous Functions (Rome, 1973), Symposia Mathematica, vol. 17, Academic Press, 1976, pp. 25-41.

$\left[\mathbf{K}_{1}\right]$ B. S. Klebanov, On the metrization of $\phi$-spaces, Soviet Math. Dokl. 20 (1979), 557-560.

$\left[\mathbf{K}_{2}\right] \ldots$, Closed and inductively closed images of products of metric spaces, Seminar on General Topology (P. S. Aleksandrov, ed.), Moskov. Gos. Univ., Moscow, 1981, pp. 114-134.

[MN] E. Michael and K. Nagami, Compact-covering images of metric spaces, Proc. Amer. Math. Soc. 37 (1973), 260-266.

$\left[\mathbf{S}_{1}\right]$ V. Šcepin, Every finite-dimensional compact absolute neighborhood retract is metrizable, Soviet Math. Dokl. 18 (1977), 402-406.

[S $\left.\mathbf{S}_{2}\right]$, Sur les applications continues des cubes de Tihonov, C.R. Acad. Sci. Paris Ser. A-B 288 (1979), 257-260.

[T] K. Tsuda, Metrizability of general ANR, Proc. Amer. Math. Soc. 96 (1986). 375-378. 